\title{
Correction to: Depressive residual symptoms are associated with illness course characteristics in a sample of outpatients with bipolar disorder
}

\author{
Gianluca Serafini ${ }^{1}$ - Gustavo H. Vazquez ${ }^{2,3,4} \cdot$ Xenia Gonda $^{5,6,7} \cdot$ Maurizio Pompili $^{8} \cdot$ Zoltan Rihmer $^{5} \cdot$ Mario Amore $^{1}$
}

Published online: 6 March 2018

○) Springer-Verlag GmbH Germany, part of Springer Nature 2018

\section{Correction to: \\ European Archives of Psychiatry and Clinical Neuroscience \\ https://doi.org/10.1007/s00406-018-0875-5}

Please, 6-7 lines before the bottom (abstract section), delete the following statement:

"Subjects with residual symptoms were less likely to report substance abuse $\left(\chi_{(2)}^{2}=11.937, p \leq 0.005\right)$ and lifetime psychotic symptoms $\left(\chi_{(2)}^{2}=10.577, p=0.005\right)$ "

and insert:

"Subjects with residual symptoms were less likely to report substance abuse $\left(\chi_{(2)}^{2}=11.937, p \leq 0.005\right)$, are more likely to report lifetime psychotic symptoms $\left(\chi_{(2)}^{2}=10.577\right.$, $p=0.005) "$

Please, 3-4 lines before the bottom (abstract section), delete the following statement:

"After multivariate analyses, a significant positive contribution to residual symptoms was given only by duration of current illness episode ( $\beta=0.003 ; p \leq 0.05)$, and lifetime psychotic symptoms $(\beta=1.094 ; p \leq 0.005)$ ".

and insert:

"After multivariate analyses, a significant positive contribution to residual symptoms was given only by duration of current illness episode ( $\beta=0.003 ; p \leq 0.005)$, and significant distressing life events in the last 6 months $(\beta=1.094 ; p$ $\leq 0.005)$ ".

Page 4, column 2, 10 lines from the top (Results section), delete the following statement:

"likely to report substance abuse (30.3 vs. 69.7\%)" and insert:

"likely to report substance abuse (46.7 vs. $53.3 \%$ )"

Finally, throughout the Discussion section, page 10, column 1, 14 lines before the bottom, delete the following statement:

"the functional outcome in BD appear to reflect". and insert:

"the functional outcome in BD appears to reflect".

The original article can be found online at https://doi.org/10.1007/ s00406-018-0875-5.

Gianluca Serafini

gianluca.serafini@unige.it

1 Department of Neuroscience, Rehabilitation, Ophthalmology, Genetics, Maternal and Child Health (DINOGMI), Section of Psychiatry, University of Genoa, IRCCS Ospedale Policlinico San Martino, Largo Rosanna Benzi 10, 16132 Genoa, Italy

2 International Consortium for Bipolar and Psychotic Disorder Research, McLean Hospital, Belmont, MA, USA

3 Department of Neuroscience, Palermo University, Buenos Aires, Argentina
4 Department of Psychiatry, Queens University, Kingston, Ontario, Canada

5 Department of Psychiatry and Psychotherapy, Kutvolgyi Clinical Center, Semmelweis University, Budapest, Hungary

6 MTA-SE Neuropsychopharmacology Research Group, Hungarian Academy of Sciences and Semmelweis University, Budapest, Hungary

7 NAP-A-SE New Antidepressant Target Research Group, Semmelweis University, Budapest, Hungary

8 Department of Neurosciences, Suicide Prevention Center, Sant'Andrea Hospital, University of Rome, Rome, Italy 